\title{
Modelling of Li-ion Batteries Dynamics using Impedance Spectroscopy and Pulse Fitting: EVs Application
}

\author{
G. Pérez, I. Gandiaga, M. Garmendia, J.F. Reynaud, U. Viscarret \\ IK4-IKERLAN, Po J. Ma Arizmendiarrieta, 2, 20500 Arrasate-Mondragón, Gipuzkoa, Spain, gperez@ikerlan.es
}

\begin{abstract}
One of the most relevant tasks that must be carried out by a Battery Management System (BMS) is the diagnosis of the battery state. An important part of the algorithms used for determining the State of Charge (SOC) or the State of Health (SOH) requires a cell model to run. The most precise is the model used, the best is the estimation achieved by the algorithm. In this paper, two techniques for obtaining a model of the cell dynamics and calculating its parameters are analyzed: the time domain characterization and the frequency domain or impedance-based characterization. Their principal characteristics and some relevant considerations to take into account are explained, as well as the obtained results. The performance of both models is compared in terms of the voltage error and the requirements to use them. Finally, a combined methodology is proposed to overcome the problems which can appear when each technique is employed. The resultant model is validated at $25^{\circ} \mathrm{C}$ in all SOC range using real measurements of a $40 \mathrm{Ah} \mathrm{Li-ion} \mathrm{cell}$ with different current profiles, including pulses of diverse lengths and FUDS driving cycles. The tests show small error between the real response of the cell and the output of the model.
\end{abstract}

Keywords: battery model, BMS, diagnosis, EV, impedance spectroscopy

\section{Introduction}

An accurate SOC determination is critical in Electric Vehicles (EVs) as an indicator of the vehicle autonomy [1]. In general, the Li-ion cell or full battery pack diagnosis is performed by the BMS as one of the main duties in conjunction with the accomplishment of protection functions.

There are many different techniques that can be used to perform an estimation of the state of a battery [2]. The adaptive or closed loop algorithms like Kalman Filtering produce a highly reliable estimation as they can mitigate the inaccuracies caused by measurement errors [3] or changing work conditions. However, this kind of algorithms requires a precise model of the cell or the battery pack to achieve an accurate estimation. In the case of EVs, the model must work with a wide variety of dynamics, depending on accelerations and decelerations.

A cell model is often composed of two main parts: a static model which relates the Open Circuit Voltage (OCV) to the SOC, and a model of the cell dynamics which describes the reactions to changes in the system inputs. In this paper, the part corresponding to the cell dynamics will be analysed. Some different cell models can be found in the literature [4-10]. Electrochemical models are based in the representation of chemical effects which occur inside the cell using equations with 
certain complexity [5, 6]. On the other hand, mathematical or electrical equivalent models represent the relationship between the inputs and outputs of a system constituted by the cell or the pack, while they do not model directly the processes that actually happen inside the cell [710]. This work is focused on electrical equivalent circuit models, because although they are simpler to compute and therefore suitable for being implemented in a BMS, their parameters can be associated with measurable effects of the cell dynamics.

Two main techniques can be used to calculate the model parameters. The first is explained in section 2 and uses time domain measurements, while the second one, detailed in section 3 , is based on frequency domain. Both techniques will be analysed and the response of the obtained models to different current profiles with a $40 \mathrm{Ah}$ NMC Li-ion cell will be shown. In section 4 a combined methodology which solves some of the problems found with the two methods explained is proposed, and in section 5 the obtained model is validated with several tests, including driving cycles. Finally, the conclusions of this work are presented in section 6 .

\section{Time domain identification}

Employing time domain measurements, the parameters of the model can be extracted applying current pulses and using the least square technique to match the response of the model with the real measurement of the cell voltage. A model based on second order Randles has been selected like in [11] and it is represented in Figure 1.

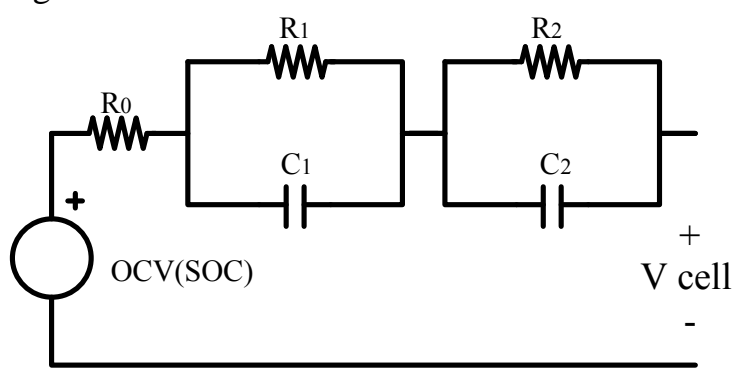

Figure 1: Second order Randles model adjusted to the temporal measurements

The voltage source represents the OCV of the cell and it is non-linearly dependent on the current SOC. This relationship is implemented by means of a look-up table.
The profile used in the adjustment of the considered model consists of five pulses of $20 \mathrm{~A}$ and $10 \mathrm{~s}$, with a $15 \mathrm{~s}$ pause between each pulse and a final relaxing period.

As the Figure 2 shows, one RC circuit is not enough to represent the response to several pulses, because it would be difficult to model properly the dynamics which appear within the pulses and the progressive decrease of voltage between each pulse. In this figure, the reduction in the OCV caused by the discharge has been compensated to appreciate the effect clearly.
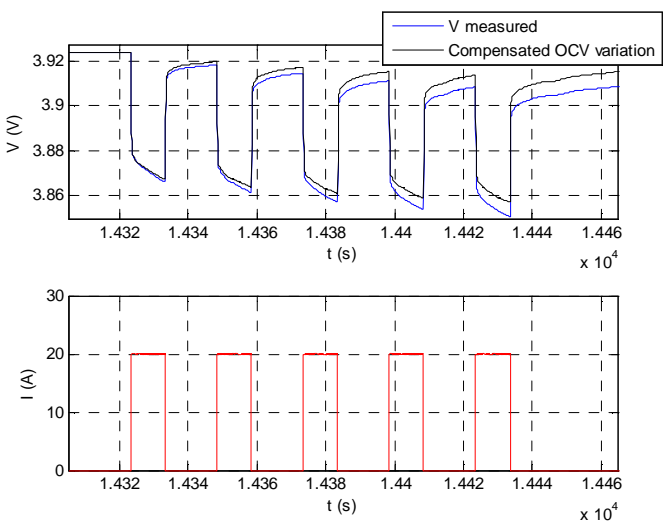

Figure 2: Cell response to $20 \mathrm{~A}, 10 \mathrm{~s}$ current discharge pulses at $25{ }^{\circ} \mathrm{C}, 80 \%$ SOC and compensation of the

OCV decrease

As it has been shown, the small changes in the SOC during the tests cause an OCV variation. Therefore, it is crucial to take into account the OCV compensation to adjust properly each parameter. For example, with the used cell, at $80 \%$ SOC, a variation of $0.8 \%$ SOC produces a voltage reduction of $4.7 \mathrm{mV}$, enough difference to invalidate the adjustment. If this OCV change is not corrected, the fitting of the parameters will be done as if there were too slow dynamics (which in fact do not exist) that produce a drop in the voltage which is not recovered in a long time. The OCV compensation can be computed with each new sample using the coulomb counting method to determine the SOC and the known relationship between itself and the OCV.

Equation (1) describes the expression of the modelled cell voltage, $V_{\text {cell }}$, discretized with sample time $T_{S}$ and the current $I$, considered positive in discharge. As the parameters of the model change with the SOC, it is necessary to repeat the fitting procedure at various SOC. 


$$
\begin{aligned}
& V_{\text {cell }}(k+1)=\operatorname{OCV}(\operatorname{SOC}(k+1)) \\
& \quad-R_{0} I(k+1)-\left[V_{R C 1}(k) e^{-\frac{T_{s}}{R_{1} C_{1}}}\right. \\
& \left.\quad+R_{1} I(k)\left(1-e^{-\frac{T_{s}}{R_{1} C_{1}}}\right)\right] \\
& -\left[V_{R C 2}(k) e^{-\frac{T_{S}}{R_{2} C_{2}}}\right. \\
& \left.\quad+R_{2} I(k)\left(1-e^{-\frac{T_{s}}{R_{2} C_{2}}}\right)\right]
\end{aligned}
$$

The response of the model fitted and the test used in the adjustment at $80 \%$ SOC are represented in Figure 3.
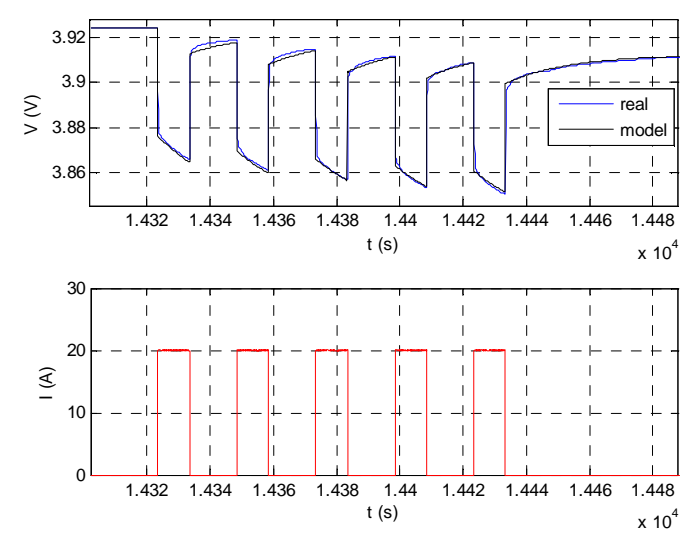

Figure 3: Measured response of the cell and output of the model

The use of the least square method presents some drawbacks. As this algorithm tries to minimize the mean least square error, this method becomes very sensitive to changes in the length of the set of samples. When slow and fast dynamics are included in the studied set, the adjustment of slow dynamics is prioritized. This is because the slow dynamics are composed by more samples, and therefore, they are more significant to reduce the Mean Square Error (MSE), according to equation (2). If the length of the set is reduced, maintaining the section where the fast dynamics are represented, and removing samples in the section of slow dynamics, they will lose importance in the final model. This effect can be appreciated when a set consisting in several pulses and a later relax period is adjusted.

$M S E=\frac{1}{T} \int_{0}^{T}\left(V_{\text {model }}(t)-V_{\text {meas }}(t)\right)^{2} d t$

Another drawback is the dependence on the width of the used pulses. The model obtained using this method represents with very low error pulses with durations similar to the pulses used in the adjustment, but it has more problems when the length of the pulses changes. The results of Figure 3 show that the adjusted model can represent precisely the time constants similar to those used in the adjustment, but the error increases significantly representing slower dynamics. In addition, the values used in the initialization of the fitting algorithm have considerable influence on the obtained results.

All this factors can produce quite variable parameters between different characterizations, making difficult the result comparison and the data interpolation to obtain a model valid in all SOC range. One possible solution requires the use of pulses of different lengths for the adjustment, to achieve modelling different dynamics accurately. Nevertheless, this option will increase the time of the process as it requires more RCs (a higher order model) to model more time constants, and it will prioritize the modelling of the slow dynamics because of the effect above explained.

The parameters of the model change with the SOC and the temperature, so it is necessary to repeat all this process several times to characterize a model in the whole range of work. Moreover, it is necessary to use a correct initialization to model all the dynamics required. Additionally, when long pulses are used, an appreciable variation in the SOC can be produced, so it is not possible to characterize the model at a fixed SOC. Another problem found is related to the measurement equipment. Some peaks can be observed in the error at the fast transitions, which occurs because the filter included in the measurement equipment smooths the real response of the cell, causing an increment in the error. Consequently, this error is neglected when comparing the performance of the model.

\section{Impedance-based model}

The impedance-based model requires performing a characterization of the frequency response of the cell. The Electrochemical Impedance Spectroscopy (EIS) permits to obtain a measurement of the cell impedance which is used to develop the cell model. At each SOC, a new EIS is carried out. The measured impedance can be modelled with equivalent electrical components $[12,13]$. In the Figure 4 the result of the EIS measurements in all SOC range and the equivalent electric components which can model the different parts of the impedance are shown. 


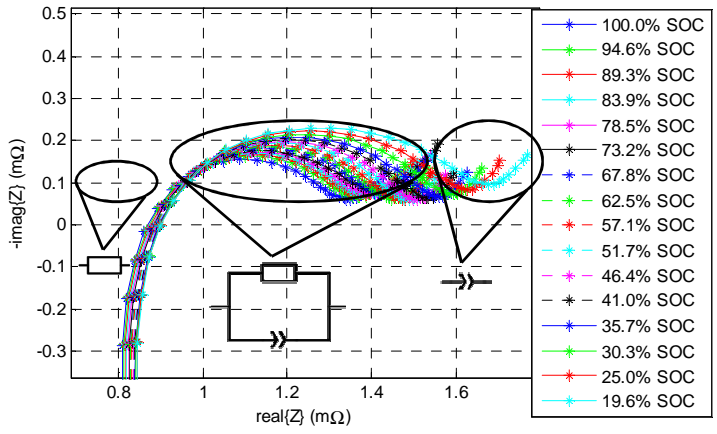

Figure 4: EIS measurements at full range of SOC at $25^{\circ} \mathrm{C}$ and representation of equivalent impedances

At high frequencies the behaviour of the impedance is mainly inductive, but this parameter is commonly neglected because it represents very fast dynamics, so the employed model is composed of resistors and Constant Phase Elements (CPEs), whose impedance is expressed in equation (3). The model described above is represented in Figure 5.

$$
Z_{C P E}=\frac{\sigma}{(j \omega)^{\alpha}}, \quad 0<\alpha<1
$$

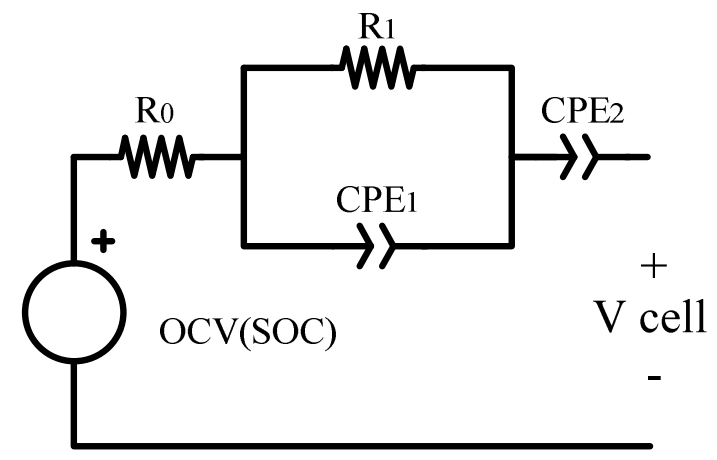

Figure 5: Impedance-based model for the EIS measurements

With the studied cell, the sub-circuit composed by one resistor $\left(\mathrm{R}_{1}\right)$ and one $\mathrm{CPE}\left(\mathrm{CPE}_{1}\right)$ in parallel represents fast dynamics which can be also neglected. It is possible to check that the time constant of an RC adjusted to these frequencies has the same magnitude than the sample time used to register the measurements. Therefore, its effect is hardly appreciated and the voltage error caused for this approximation is very low. Hence, the final model employed consists of one CPE and one resistor, which includes the resistive part of the impedance $R_{0}$ and the resistor $R_{1}$ of the parallel sub-circuit that has been neglected.
These two elements can be easily adjusted applying complex least squares, fitting the equation of the modelled impedance described in equation (4) to the impedance data measured in each EIS at the frequencies where the effect of the $\mathrm{CPE}$ is predominant.

$$
Z=R+\frac{\sigma}{(j \omega)^{\alpha}}
$$

where $R=R_{0}+R_{1}$.

The expression of the CPE is a fractional equation that cannot be simulated easily in time domain, so its impedance is approximated using RC subcircuits. The modelled impedance represents more accurately the measured impedance when more sub-circuits are used. The main difference between the time domain model and this model is that in this case the RCs are calculated adjusting the measured impedance instead of the time response of the cell.

This approximation of the impedance allows performing the time domain simulation of the model for any input current. As the difference equations of the RCs are well known, it is possible to calculate the cell response in real time, and even to compute it in a BMS.

The final model is composed by one resistor $(R)$ and one CPE, which is approximated with several RC sub-circuits, as it is shown at Figure 6.

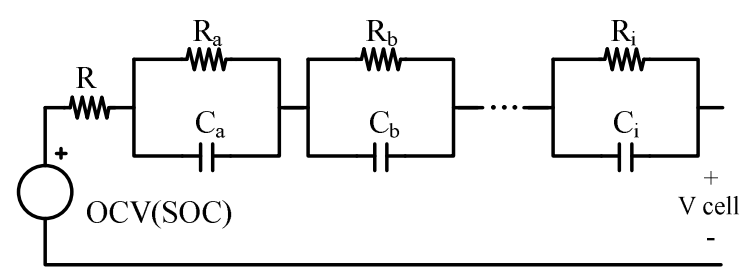

Figure 6: Modelled impedance of the cell

The main advantage of this method is that it is relatively easy to obtain an enough high order model to represent accurately a wide range of dynamics. Thereby, this technique can model the slow dynamics without increasing the error with the fast ones, fixing the most important problem that has been found using the time domain characterization.

Furthermore, the obtained parameters with different EIS measurements have more stability as they present less variability. Their progressive 
change with the SOC allows calculating a model valid at intermediate SOC values, in which the EIS has not been performed, using linear interpolation.

All these characteristics make this model more general and suitable for reproducing the response of the real cell to pulses with different lengths and in all SOC range.

The main problem found using this method appears when the EIS equipment and the cell are disconnected between different measurements, which can produce a significant variation in the real part of the impedance, corresponding with a change in the resistance expressed as a horizontal displacement of the curves in the Nyquist diagram. This causes difficulties to compare EIS measurements in different conditions. The displacement can be reduced using a careful setup, although some variation is unavoidable. The technique proposed in the next section allows the correction of the measurements to make them more consistent.

\section{Combined methodology}

A combined methodology is proposed to overcome some of the problems found with the previous techniques. This methodology requires the performance of EIS measurements and pulse current tests. The test is repeated over all the SOC range, as it was done before. At each selected SOC, an EIS is carried out after a period of relaxation of the cell. Then, when the cell is relaxed again, several discharge pulses are extracted, before starting a new discharge to reach the next SOC where the procedure is going to be performed. The current pulses employed are defined in the same way explained in the section 2. These pulses consume little energy, enough to not produce a relevant change in the SOC which could affect to the cell dynamics varying the parameters of the model, although sufficient information to accomplish two tasks can be gathered. The first task is the validation of the model fitted to the impedance measured in the frequency domain at that SOC, checking that it can reproduce the temporal effects with low error. The second task is the correction of the resistance $R$ in case of reconnections of the measurement equipment. It is possible to make the correction just comparing the instant voltage drop obtained with the pulses performed after the EIS, with other pulses performed with the new connection. This correction significantly affects to the response of the model. As an example, a little variation of only $0.6 \mathrm{~m} \Omega$ provokes a difference of $12 \mathrm{mV}$ when the current is $20 \mathrm{~A}$.

\section{Model validation}

The model obtained with each methodology has been validated calculating its response to a certain current profile and comparing it with the real response of the cell. In these tests a NMC Li-ion $40 \mathrm{Ah}$ cell has been used. Some of the results obtained with the impedance-based model adjusted using the combined methodology are represented in the following figures. The current profiles are composed by pulses from $10 \mathrm{~s}$ to $900 \mathrm{~s}$ and FUDS driving cycles.

Figure 7 represents the model response to $20 \mathrm{~s}$ pulses and Figure 8 the response to $900 \mathrm{~s}$ pulses. Figure 9 and Figure 10 show the response of the model to several FUDS cycles over a wide range of SOC, which is depicted in Figure 11. These results show small error (less than $8 \mathrm{mV}$ with the pulses and $12 \mathrm{mV}$ in the driving cycles) in the voltage estimation and in its equivalent SOC error. As it has been explained in section 2, some peaks in the error appear when the current has abrupt transitions, because the filter of the measurement equipment smooths the response of the cell. Accordingly, these peaks should not be taken into account. In the results corresponding to current pulses they have been deleted to achieve a clearer representation of the results. However, the tests with FUDS cycles are composed entirely of steps in the current profile so the suppression of this effect is not feasible.

The error plot of the FUDS test (Figure 9) shows an offset which increases the global error. This offset remains even when the cell is relaxed, so it is not caused by the error of the model of the cell dynamics. Thus, it is caused by an error determining the OCV. The principal reason is that the effect of the hysteresis on the OCV has not been modelled, and this voltage has been supposed approximately equal to the discharge boundary of the OCV. The hysteresis effect on this cell is not very pronounced in comparison with other chemistries (e.g. $\mathrm{LiFePO}_{4}$ ), but it can make a difference of $6 \%$ SOC between the charge and the discharge OCV's boundaries at the same voltage. This error can be reduced employing a more complex model of the OCV-SOC relationship including the hysteresis effect. 

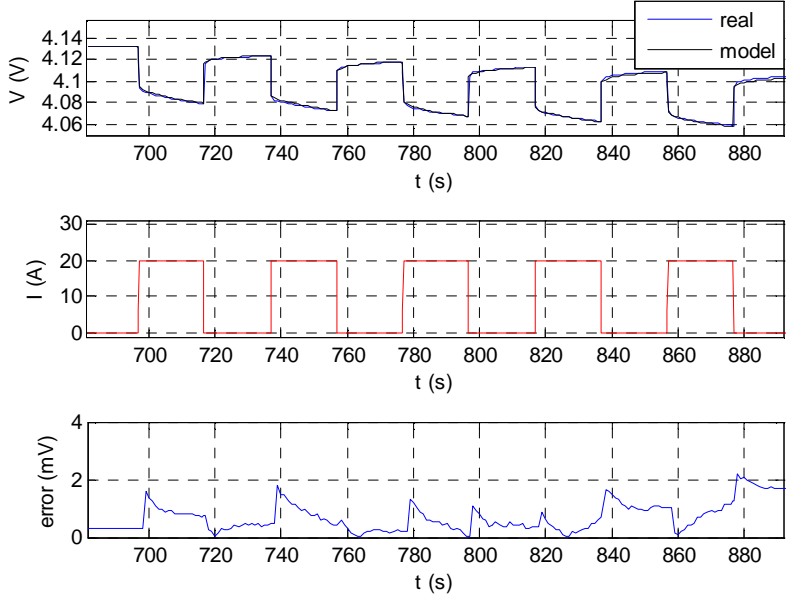

Figure 7: Impedance-based model response with 20 s, 20 A discharge pulses at $25^{\circ} \mathrm{C}$ and $100 \%$ SOC
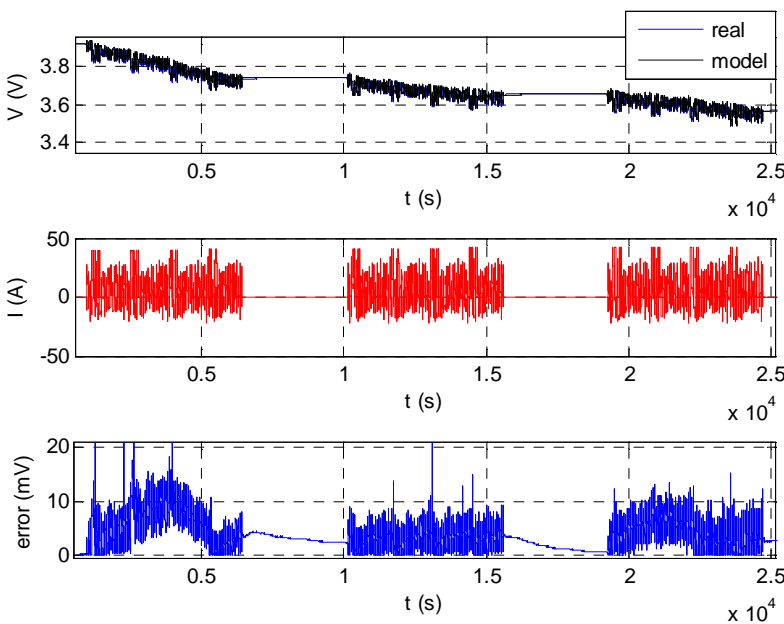

Figure 9: Impedance-based model response and voltage error with FUDS cycles at $25^{\circ} \mathrm{C}$
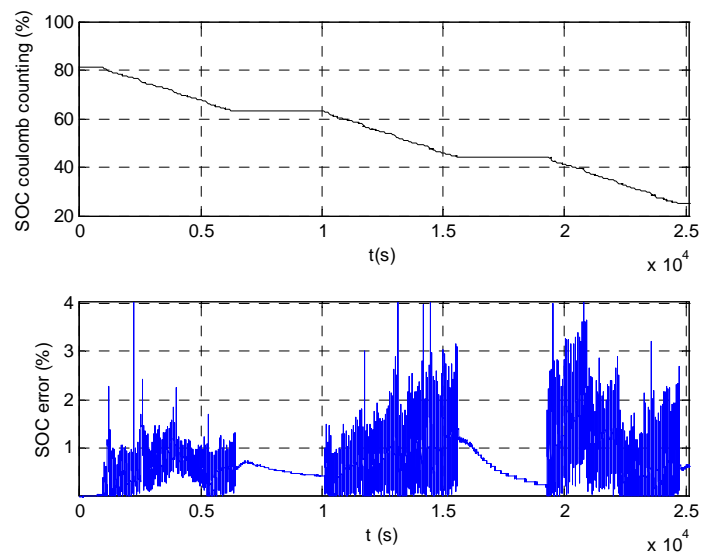

Figure 11: Real SOC and equivalent SOC error during FUDS cycles at $25^{\circ} \mathrm{C}$
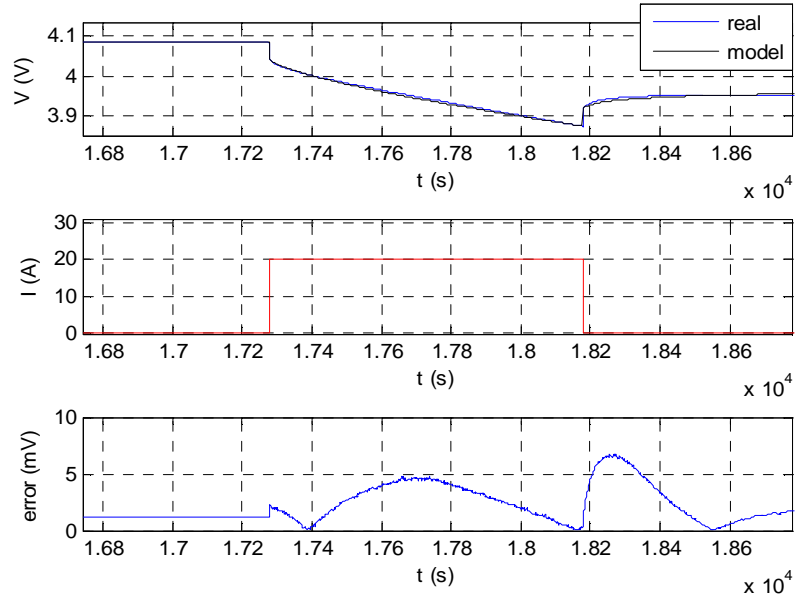

Figure 8: Impedance-based model response with a $900 \mathrm{~s}$, 20 A discharge pulses at $25{ }^{\circ} \mathrm{C}$ and $95 \%$ SOC
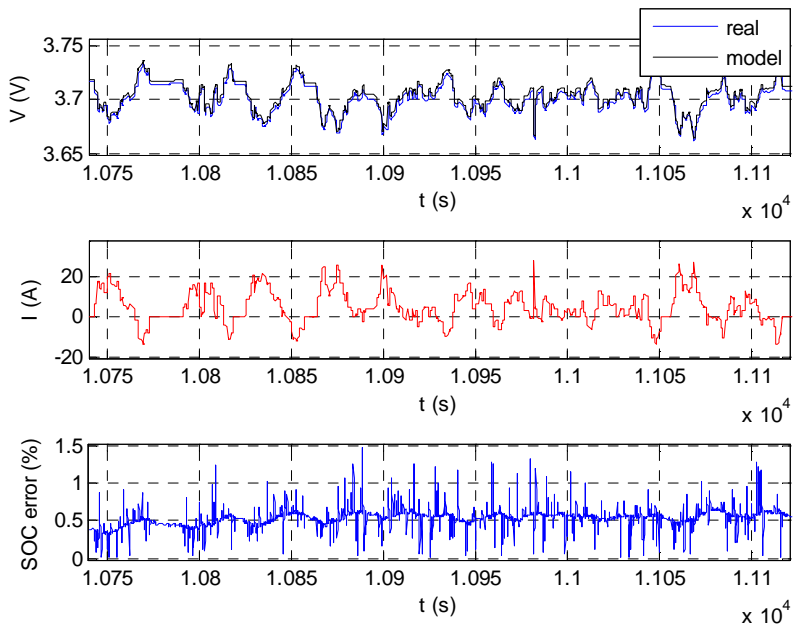

Figure 10: Zoom of the model response and equivalent SOC error in a FUDS cycle

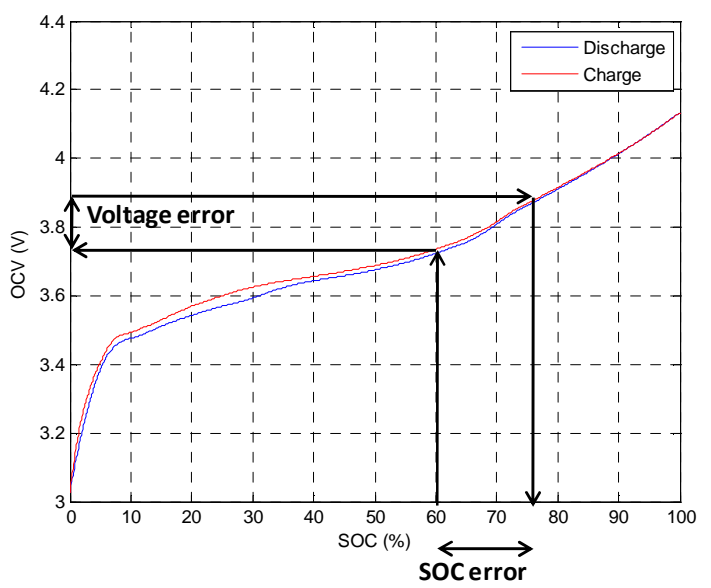

Figure 12: OCV-SOC relationship and calculation of the equivalent SOC error 
In Figure 10 and Figure 11 the equivalent SOC error is represented. It is an approximation of the real SOC error when the model is used to determine the SOC of the cell with the OCV, which is calculated using the response of the model and the measurement of the cell voltage. The value of the equivalent SOC error is obtained from the voltage error (difference between the model output and the voltage measured), because it is also the error on the OCV determination in open loop. As it is shown in Figure 12, the OCV at the current SOC is obtained, and the voltage error is added to this value. The new voltage is transformed again into SOC to calculate the error as the difference between this SOC and the current SOC.

\section{Conclusions}

Two different methodologies for identifying cell model parameters have been analyzed. The frequency based method presents less variability in the obtained parameters, and it has been selected to generate a model valid in all SOC range and capable of simulating a wide variety of dynamics. A combined methodology has been proposed to take advantage of the strength of both methods and solve problems which appear when one of the basic methods is used. The main advantages and disadvantages of each technique are summarized in Table 1.

The performance of the model has been checked using different current profiles like pulses or FUDS driving cycles, showing little error in the simulated voltage. The model is suitable to be employed in an algorithm to determine the cell SOC in open or closed loop, and can be implemented in a BMS. This model allows to estimate the OCV without waiting until the cell is relaxed, and the obtained OCV can be used to perform the determination of the SOC or to correct the value obtained with another method as coulomb counting. The low equivalent SOC error shows that this model can be used effectively to get an accurate estimation.

The future steps to improve and complete the model will be the introduction of a hysteresis model to reduce the error in the OCV-SOC relationship, and the definition of a methodology to extend the model to work at varying temperature.

Table 1: Summary of the advantages and disadvantages of each method for obtaining the cell model

\begin{tabular}{|c|c|c|c|}
\hline Method: & Time domain & Frequency domain & Combined \\
\hline 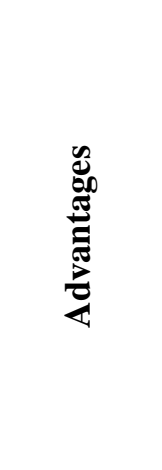 & $\begin{array}{l}\text { - Very low error in adjusted } \\
\text { test } \\
\text { - Easy to obtain the } \\
\text { parameters with standard } \\
\text { techniques like least } \\
\text { squares }\end{array}$ & $\begin{array}{l}\text { - Easy to adjust a higher order } \\
\text { model } \\
\text { - Works in a wider range of } \\
\text { frequencies with low error } \\
\text { - Allows direct interpolation } \\
\text { to achieve a model valid in } \\
\text { all SOC range }\end{array}$ & $\begin{array}{l}\text { - Easy to adjust a higher order } \\
\text { model } \\
\text { - Works in a wider range of } \\
\text { frequencies with low error } \\
\text { - Allows direct interpolation } \\
\text { to achieve a model valid in } \\
\text { all SOC range } \\
\text { - Ability to correct } \\
\text { displacement of the curves } \\
\text { caused by changes in } \\
\text { connections }\end{array}$ \\
\hline 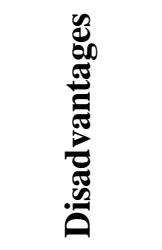 & $\begin{array}{l}\text { - High error with pulses of } \\
\text { different dynamics } \\
\text { - Difficult to adjust a higher } \\
\text { order model }\end{array}$ & $\begin{array}{l}\text { - Need of performing EIS } \\
\text { (specific equipment } \\
\text { required) } \\
\text { - High dependence of } \\
\text { connections }\end{array}$ & $\begin{array}{l}\text { - Need of performing EIS } \\
\text { (specific equipment } \\
\text { required) }\end{array}$ \\
\hline
\end{tabular}




\section{Acknowledgments}

This work was supported by Etortek program of the Basque Government.

\section{References}

[1] J. Xu et. Al., State of charge estimation online based on EKF-Ah method for lithium-ion power battery, Proceedings of the 2009 2nd International Congress on Image and Signal Processing, CISP'09, $\mathrm{n}^{\circ} 5303451$.

[2] V. Pop et. Al., State of the art of battery state of charge determination, Measurement Science and Technology, 16(2005), 93-110.

[3] S. Lee et. Al., State-of-charge and capacity estimation of lithium-ion battery using a new open-circuit voltage versus state-ofcharge, Journal of Power Sources, 185(2008), 1367-1373.

[4] I.S. Kim, The novel state of charge estimation method for lithium battery using sliding mode observer, Journal of Power Sources, 163(2006), 584-590.

[5] A. P. Schmidt et. Al., Model-based distinction and quantification of capacity loss and rate capability fade in Li-ion batteries, Journal of Power Sources, 195(2010), 7634-7638.

[6] V. Agarwal et. Al., Development and validation of a battery model useful for discharging and charging power control and lifetime estimation, IEEE Transactions on Energy Conversion, 25(2010), 821-835.

[7] C. Zhang, et. Al., Identification of dynamic model parameters for lithium-ion batteries used in hybrid electric vehicles, High Technology Letters, 16(2010), 6-12.

[8] J. Chiasson et. Al., Estimating the state of charge of a battery, Proceedings of the 2003 American Control Conference, (2003), 2863-2868.

[9] M. Knauff et. Al., A platform for the testing and validation of dynamic battery models, 2009 IEEE Electric Ship Technologies Symposium, (2009), 554-559.
[10] R. C. Kroeze et. Al., Electrical battery model for use in dynamic electric vehicle simulations, 2008 IEEE Power Electronics Specialists Conference, (2008), 1336-1342.

[11] M. Dubarry et. Al., Development of a universal modeling tool for rechargeable lithium batteries, Journal of Power Sources, 174(2007), 856-860.

[12] D. Andre et. Al., Characterization of highpower lithium-ion batteries by electrochemical impedance spectroscopy. II: Modelling, Journal of Power Sources, 196(2011), 5356-5363.

[13] M. Montaru et. Al., Frequency and temporal identification of a Li-ion polymer battery model using fractional impedance, Oil and Gas Science and Technology, 65(2010), 6778.

\section{Authors}

Gustavo Pérez Rodríguez was born in 1987 in Oviedo (Spain). He received the M.Sc. degree in Telecommunication Engineering from the University of Oviedo in 2011. He is currently working toward the Ph.D. degree in the Power Electronics and Energy Storage Systems Area, IK4IKERLAN Technology Research Centre in collaboration with the University of Mondragón (Spain). His research interests include battery management systems, energy storage, cell modelling and diagnosis algorithms (SOC/ SOH).

Iñigo Gandiaga Ondaro was born in 1986 in Durango (Spain). He received a Diploma in Physics from the University of the Basque Country, UPV-EHU (Spain) in 2010. He joined the Alternative Generation Unit of IK4-IKERLAN Technology Research Centre, Mondragón (Spain), in 2010.

His research interests include energy storage technologies, particularly $\mathrm{SOC} / \mathrm{SOH}$ estimation algorithms for lithium-ion Battery Management Systems and battery storage systems sizing methodologies. 


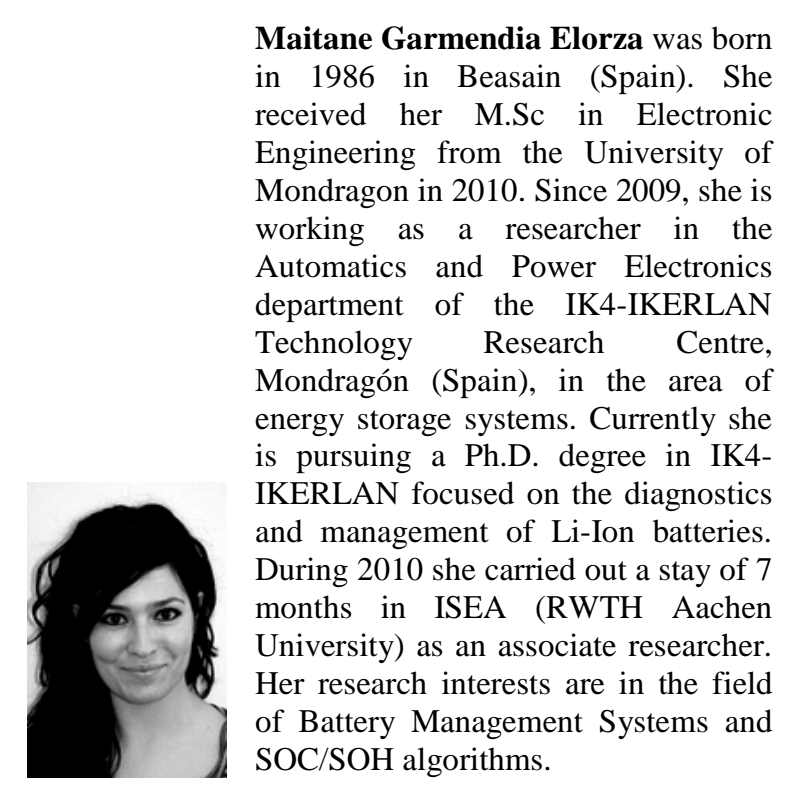

Jean-François Reynaud received B.Sc. and M.Sc degrees in Automatics and Industrial Electronics from Toulouse University (France) and Tarragona University (Spain), and the Ph.D. degree in electrical engineering from Toulouse University, in 2006 and 2011 respectively. His field of research lies in the development of new power electronics and battery management systems for lithium-ion batteries, including advanced algorithms in SOC and $\mathrm{SOH}$ determination and battery pack integration. He is currently working as

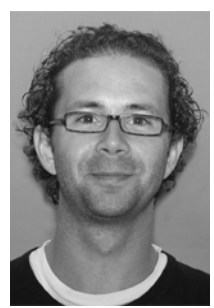
a research engineer in IK4-IKERLAN in the product development department in the field of energy storage, grid-scale and transportation applications (renewable integration, elevators, railway...). $\mathrm{He}$ is author/coauthor of various journal and conference papers.
Unai Viscarret received the B.Sc degree in Electronics from the University of Mondragón, Mondragón, Spain in 1998, and the M.Sc degree from the Swiss Federal Institute of Technology Lausanne (EPFL), Lausanne, Switzerland, in 2001.

Since 2001 he has been workings as a researcher for the IK4-IKERLAN Technology Research Centre, Spain. His research interests include the application of power electronics devices to the distribution network for

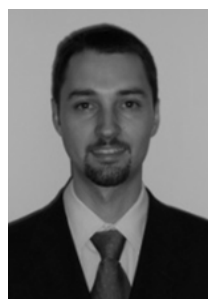
power quality improvement and distributed generation interfaces, as well as advanced control strategies for medium voltage traction converters. During the last years he is working actively on designing advanced storage systems (particularly Lithium Ion batteries). 